\title{
Effect of nitrogen gas on preparation of Ti-Al-N thin films by pulsed laser ablation
}

\author{
Akiharu Morimoto ${ }^{1}$, Hideki Shigeno ${ }^{2}$, Shinya Morita, Yasuto Yonezawa ${ }^{3}$, Tatsuo Shimizu \\ Department of Electrical and Computer Engineering, Kanazawa University, \\ Kodatsuno, Kanazawa 920-8667, Japan
}

\begin{abstract}
The effect of ambient $\mathrm{N}_{2}$ gas on the preparation of $\mathrm{Ti}_{0.9} \mathrm{Al}_{0.1} \mathrm{~N}$ (TAN) thin films for ferroelectric capacitors by pulsed laser ablation (PLA) using ArF and $\mathrm{KrF}$ excimer lasers was explored. The TAN films were prepared on (100)Si substrates heated at $620{ }^{\circ} \mathrm{C}$ in various $\mathrm{N}_{2}$ pressures ranging from vacuum to $130 \mathrm{~Pa}$. The TAN crystal growth was found to be influenced by the content of unintentionally-incorporated $\mathrm{O}$ which was found to be controlled by the introduction of $\mathrm{N}_{2}$ gas into the deposition chamber. The $\mathrm{O}$ content for films prepared by $\mathrm{KrF}$ was found to be smaller than that for films prepared by ArF because of the smaller optical absorption cross-section of $\mathrm{KrF}$ excimer laser for residual $\mathrm{O}_{2}$ or $\mathrm{H}_{2} \mathrm{O}$ molecules in the chamber and/or the higher deposition rate. The TAN film prepared by $\mathrm{KrF}$ excimer laser was found to be nearly epitaxial Si with a cube-on-cube crystallographic orientation.
\end{abstract}

\footnotetext{
1 Corresponding author: Tel +81-76-234-4876, Fax +81-76-234-4900,

amorimot@ec.t.kanazawa-u.ac.jp

2 Present address: Shin-Etsu Handotai Co., Ltd., 2-13-1 I sobe, Annaka, Gunma 379-01, J apan

3 On leave from the Industrial Research Institute of I shikawa, Tomizu, Kanazawa 920-0223, J apan
} 
PACS: $\quad$ 61.10.-i, 72.15.-v, 81.10.-h

Keywords: Ti-Al-N electrode film, PZT capacitors, Si substrate, PLD, nitrogen ambient gas, oxygen impurity 


\section{Introduction}

$\mathrm{Pb}(\mathrm{Zr}, \mathrm{Ti}) \mathrm{O}_{3}(\mathrm{PZT})$ ferroelectric capacitors is a material employed to fabricate nonvolatile memory and requires oxidation-resistant electrodes. Pt is conventionally used as an electrode, but it creates fatigue when used with PZT[1], Pt hillocks form[2], the Pb loss is found to be from the PZT[3], and $\mathrm{Pt}$ reduces $\mathrm{PbO}$ in the presence of $\mathrm{H}_{2}$ through a catalytic reaction[4]. TiN has been extensively studied for the diffusion barrier in advanced integrated-circuit devices, suggesting that TiN can be introduced without any difficulties into the present or future LSI process[5]. TiN, however, is not sufficiently oxidation-resistant[6] for the preparation of PZT. Thus, we proposed Ti-Al-N (TAN) as an oxidation-resistant electrode material for the PZT based on the idea that aluminum oxide is good barrier against oxidation[7]. Perovskite PZT film was found to be grown on the TAN electrode, resulting in a ferroelectric hysteresis loop. However, the interface layer of the TAN was oxidized to some extent by the PZT deposition due to a low quality of the TAN film[7]. In the present study, the effect of ambient $\mathrm{N}_{2}$ gas on the preparation of TAN thin films by PLA is explored for high-quality film synthesis. Especially, from the viewpoint of the collision of depositing particles, including atoms, molecules, and clusters, with ambient gas molecules, the effect of ambient gas was examined.

\section{Experimental}

TAN films were deposited using ArF and the KrF excimer lasers (Shibuya ES5000) with wavelengths of $193 \mathrm{~nm}$ and $248 \mathrm{~nm}$, respectively. The lasers were operated at a pulse repetition rate of 5 or $10 \mathrm{~Hz}$. The fluence was $4.2 \mathrm{~J} / \mathrm{cm}^{2}$ for the ArF laser and $6.2 \mathrm{~J} / \mathrm{cm}^{2}$ for the $\mathrm{KrF}$ laser. Two 
hot-pressed $\mathrm{Ti}_{0.9} \mathrm{Al}_{0.1} \mathrm{~N}$ targets were used; densities of $60 \%$ (low density: LD) and $91 \%$ (high density: HD) of ideal one. TAN films were prepared on (100)Si substrates heated at $620{ }^{\circ} \mathrm{C}$ in various $\mathrm{N}_{2}$ pressures ranging from vacuum to $130 \mathrm{~Pa}$. Prior to film deposition, the chamber was evacuated to about $2 \times 10^{-4} \mathrm{~Pa}$ using a diffusion pump. So, the present vacuum means the pressure of the residual gas of $2 \times 10^{-4} \mathrm{~Pa}$. The Si substrates were cleaned in a solution of HF, distilled water, and ethanol in the ratio of 1:1:10 to obtain a hydrogen-terminated Si surface. The KrF films are thicker than the ArF films by a factor of about two due to the difference in deposition rates.

\section{Results and Discussion}

3-1. TAN films prepared by ArF excimer laser with high and low density targets

Figure 1 shows the X-ray diffraction (XRD) patterns for films prepared on Si substrates by $\mathrm{ArF}$ excimer laser ablation at various $\mathrm{N}_{2}$ pressures. Only the (200) diffraction peak of the TAN structure is observed for films prepared in vacuum and 1.3 $\mathrm{Pa}$ of $\mathrm{N}_{2}$ gas. The peak is not observed when the $\mathrm{N}_{2}$ pressure exceeds $13 \mathrm{~Pa}$. This result suggests that highly [200]-oriented TAN films can be deposited in low $\mathrm{N}_{2}$ pressure. Figure 2 shows the peak intensity of the (200) diffraction as a function of the $\mathrm{N}_{2}$ pressure. In this figure, two sets of TAN films prepared using the LD and HD targets are compared. It was found that the crystal growth is enhanced by introducing $\mathrm{N}_{2}$ gas into the deposition chamber while excess $\mathrm{N}_{2}$ pressure suppresses the crystal growth. The XRD rocking curve measurement on the (200) diffraction revealed that the full width at half maximum (FWHM) for the films prepared with the $\mathrm{HD}$ target is decreased from $4.5^{\circ}$ to $3.1^{\circ}$ by increasing the $\mathrm{N}_{2}$ pressure from vacuum to $1.3 \mathrm{~Pa}$.

Fig. 2 also suggests that the HD target enhances the crystal growth, when compared with 
films formed from the LD target. The FWHM of the XRD rocking curve for the films prepared using the HD target was also smaller than that for the films prepared with the LD target. The use of the HD target in PLA may reduce the thickness of the molten layer as a consequence of more intense laser absorption in the HD target than in the LD target. This increases the temperatures of the molten layer of the target surface and the temperature of the ejected clusters, and/or increases the kinetic energy of depositing particles due to the PLA explosion[8].

The film composition was evaluated by electron-probe microanalysis (EPMA). The ratio of $\mathrm{Al}$ to $\mathrm{Al}+\mathrm{Ti}$ was found to be approximately $10 \%$ and nearly the same as the ratio in the target. Figure 3 shows the $\mathrm{O}$ content as a function of the $\mathrm{N}_{2}$ pressure. It was found that the $\mathrm{O}$ content of the deposited films is large and ranges from 30 to $40 \%$ for the films prepared using the HD target although the evaluation of the TiN composition by EPMA includes some ambiguity because of the overlapping of the $\mathrm{N} \mathrm{K}_{\alpha}$ and $\mathrm{Ti} \mathrm{L}_{1}$ lines[9]. The presence of a large amount of $\mathrm{O}$ was also confirmed by X-ray photoelectron spectroscopy (XPS). Despite the large O content, XRD spectra revealed a TAN crystal with a single phase. The EPMA measurement revealed that the $\mathrm{O}$ content decreased with increasing $\mathrm{N}_{2}$ gas to $1.3 \mathrm{~Pa}$, and then increased by further pressure increase to $130 \mathrm{~Pa}$. This minimum in Fig.3 is well correlated with the (200) peak intensity as a function of the $\mathrm{N}_{2}$ pressure. In other words, the TAN crystal growth seems to be governed by the $\mathrm{O}$ impurity content.

The dependence of the (200) diffraction intensity on the $\mathrm{N}_{2}$ pressure might be explained as follows. In ultrahigh vacuum TAN particles ejected from the target might be expected to evolve some $\mathrm{N}$ atoms during the flight process, resulting in a $\mathrm{N}$-deficient film. For instance, AlN is thermally decomposed above $2400{ }^{\circ} \mathrm{C}$ into $\mathrm{Al}$ metal and $\mathrm{N}_{2}$ gas[10]. In the present vacuum, there exist residual gases consisting of $\mathrm{H}_{2} \mathrm{O}$ and $\mathrm{O}_{2}$ gases with a background pressure of $2 \times 10^{-4} \mathrm{~Pa}$. There is a possibility that the TAN particles with $\mathrm{N}$ deficiency react with the ambient oxidation gas 
during the flight process, resulting in the oxi-nitride. In this deposition condition, if $\mathrm{N}_{2}$ gas with a pressure far larger than the residual oxidation gas is present, ejected TAN particles collide with $\mathrm{N}_{2}$ rather than $\mathrm{O}_{2}$ with a higher probability, thus reducing the $\mathrm{N}$ deficiency and the $\mathrm{O}$ impurity. Excess amount of the ambient $\mathrm{N}_{2}$ causes cooling or deactivation of the depositing particles due to excess collisions with $\mathrm{N}_{2}$. Besides, the excess $\mathrm{N}_{2}$ also causes the oxidation of depositing particles due to the excess collisions with $\mathrm{N}_{2}$ and oxidation gases. These mechanisms probably lead to the serious degradation of the TAN crystal.

Only for the TAN-resistivity measurement, (100)MgO substrates are used because of the rather low resistivity of the Si substrate. Resistivity of the TAN films increases with an increase in the $\mathrm{N}_{2}$ pressure up to $130 \mathrm{~Pa}$, from $160 \mu \Omega \cdot \mathrm{cm}$ to $10 \mathrm{~m} \Omega \cdot \mathrm{cm}$. Minimum resistivity of $160 \mu \Omega \cdot \mathrm{cm}$ was obtained in vacuum, and the resistivity was increased to be about $200 \mu \Omega \cdot \mathrm{cm}$ by $\mathrm{N}_{2}$ gas up to $1.3 \mathrm{~Pa}$.

Figure 4 shows the photographs of the scanning electron microscopy (SEM) for the films prepared in various $\mathrm{N}_{2}$ pressures. The formation of droplets was found to be largely increased by the $\mathrm{N}_{2}$ pressure of $130 \mathrm{~Pa}$ while there was no appreciable change up to $13 \mathrm{~Pa}$. This suggests that the film deposition was governed by the cluster deposition rather than the atomic or molecular deposition. In vacuum or low $\mathrm{N}_{2}$ pressure, clusters, including droplets, ejected from the target coalesce into the film because they are still sufficiently "hot". However, they may not coalesce into the film under the high pressure condition because of the increased collisional cooling of depositing clusters. This may explain the remaining of droplets on the film surface as the $\mathrm{N}_{2}$ pressure is increased.

3-2. TAN films prepared by $\mathrm{KrF}$ excimer laser with the HD target 
Figure 5 shows the XRD intensity of the (200) diffraction of TAN normalized by the film thickness as a function of $\mathrm{N}_{2}$ pressure for the $\mathrm{KrF}$ films. For comparison, data for ArF films are also shown. Comparison of the (200) intensity for the films with a laser repetition rate of $10 \mathrm{~Hz}$ and at $\mathrm{N}_{2}$ pressure of $1 \mathrm{~Pa}$ shows that the crystal growth is enhanced by using $\mathrm{KrF}$ excimer laser instead of ArF excimer laser. Furthermore, the $\mathrm{O}$ content in the $\mathrm{KrF}$ films grown at 5 and $10 \mathrm{~Hz}$ was found to be reduced to be about $20 \%$, which is smaller than that in the ArF films grown under the similar condition. The low oxygen content in the $\mathrm{KrF}$ film might be explained in terms of the low optical absorption cross-section of $\mathrm{KrF}$ excimer laser for the residual $\mathrm{H}_{2} \mathrm{O}$ or $\mathrm{O}_{2}$ molecules, and/or the high deposition rate.

From Fig. 5 the reduction of the repetition rate was found to suppress the crystal growth, contrary to what is observed in other deposition schemes. It is known that the crystal growth is enhanced by lowering the deposition rate. This opposite behavior might be explained by the cluster-deposition which was discussed earlier. In this model, the surface crystal- growth is enhanced by the high temperature of the molten layer on the substrate due to the subsequent deposition of hot clusters. Figure 6 shows X-ray pole figure for the $\mathrm{KrF}$ film using the TAN (202) plane. This figure revealed that the normal direction of this plane is directed to $\Psi=45^{\circ}$, and this plane has four-fold rotational symmetry without any trace of other orientations although some fluctuation can be seen along the direction of $\Phi$. This result suggests that the TAN film is nearly epitaxially grown on Si substrate having cube-on-cube crystallographic orientation.

\section{Conclusions}

The TAN crystal growth was found be influenced by the content of $\mathrm{O}$ unintentionally-incorporated which can be controlled by the introduction of $\mathrm{N}_{2}$ gas. The $\mathrm{O}$ content 
for the $\mathrm{KrF}$ film was found to be smaller than that for the ArF films because of the smaller optical absorption cross-section of $\mathrm{KrF}$ excimer laser for residual $\mathrm{O}_{2}$ or $\mathrm{H}_{2} \mathrm{O}$ molecules and/or the higher deposition rate. The $\mathrm{KrF}$ film was found to be nearly epitaxially grown on Si substrate with cube-on-cube crystallographic orientation.

\section{Acknowledgements}

The authors thank Prof. M. Kumeda for helpful discussions. We acknowledge K. Nagai for helping the experiments and Shibuya Kogyo Co., Ltd. for supplying the excimer laser. We also acknowledge K. Shiratsuyu of Murata Mfg. Co., Ltd. for measurement of the X-ray pole figure. The present work was supported in part by the Grant-in-Aid for Developmental Scientific Research (C) No. 0865008 from the Ministry of Education, Science, Sports, and Culture of Japan.

\section{References}

[1] T. Mihara, H. Watanabe, and C. A. Araujo: Jpn. J. Appl. Phys. 33 (1994) 3996.

[2] R. Bruchhaus, D. Pitzer, O. Eibl, U Scheithauer, and W. Hoesler, Mater. Res. Soc. Symp. 243 (1992) 123.

[3] K. Abe, H. Tomita, M. Imai, and Y Yokote, Jpn. J. Appl. Phys. 30 (1991) 2152.

[4] H. Miki, K. Kushida-Abdelghafar, K. Torii, and Y Fujisaki, Jpn. J. Appl. Phys. 36 (1997) 1132.

[5] S. Sobue, S. Mukainakano, Y. Ueno and T. Hattori: Jpn. J. Appl. Phys. 34 (1995) 987.

[6] M. Wittmer, J. Noser and H. Melchior: J. Appl. Phys. 52 (1981) 6659.

[7] A. Morimoto, Y. Yamanaka, and T. Shimizu, Jpn. J. Appl. Phys. 35 (1996) L227.

[8] S. Otsubo, T. Minamikawa, Y. Yonezawa, A. Morimoto, and T. Shimizu, Jpn. J. Appl. Phys. 29 
(1990) L73.

[9] M. Kusano, K. Tsukakoshi, Y. Taniuchi, K.Onoe, S. Misawa, and S. Tsukahara, Shinku 37 (1994) 237(in Japanese).

[10] J. K. Lumpp, and S. D. Allen, J. Mater. Res. 12 (1997) 218. 


\section{FIGURE CAPTIONS}

Fig. 1. XRD patterns for TAN films prepared on (100)Si substrates using the HD target by ArF excimer laser at various $\mathrm{N}_{2}$ pressures. Small peak around 33 deg is the diffraction from $\mathrm{Si}(200)$ plane.

Fig. 2. Peak intensity of the (200) diffraction as a function of the $\mathrm{N}_{2}$ pressure for films prepared on Si substrates by ArF excimer laser. LD and HD represent the low density and the high density targets, respectively.

Fig. 3. O content as a function of the $\mathrm{N}_{2}$ pressure for TAN films prepared on $\mathrm{Si}$ substrates using the low and high density targets by ArF excimer laser.

Fig. 4. SEM photographs for films prepared on Si substrates using the high density target by ArF excimer laser in various $\mathrm{N}_{2}$ pressures.

Fig. 5. XRD intensity of the TAN (200) diffraction normalized by the film thickness as a function of $\mathrm{N}_{2}$ pressure for the films prepared by $\mathrm{KrF}$ excimer laser. For comparison, data for ArF excimer laser are also shown.

Fig. 6. X-ray pole figure for the film prepared on the Si substrate using the high density target by KrF excimer laser using the TAN (220) plane. 
Total: 2,300 words +200 words $\mathrm{x} 6$ figures $=3,500$ words ; limits: 3,500 words $/ 6$ printed pages 


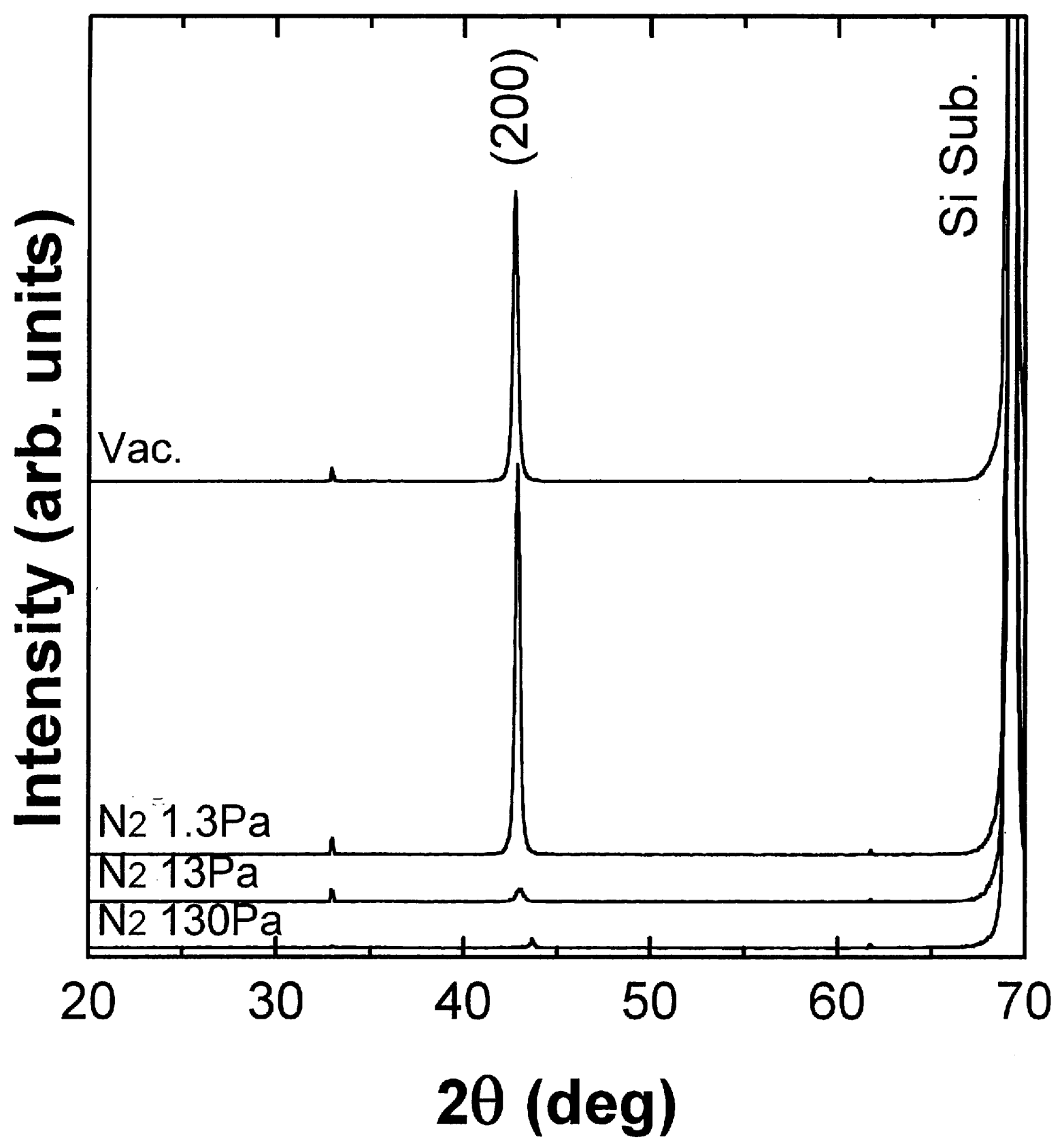

Fiq.I A Morimoto 


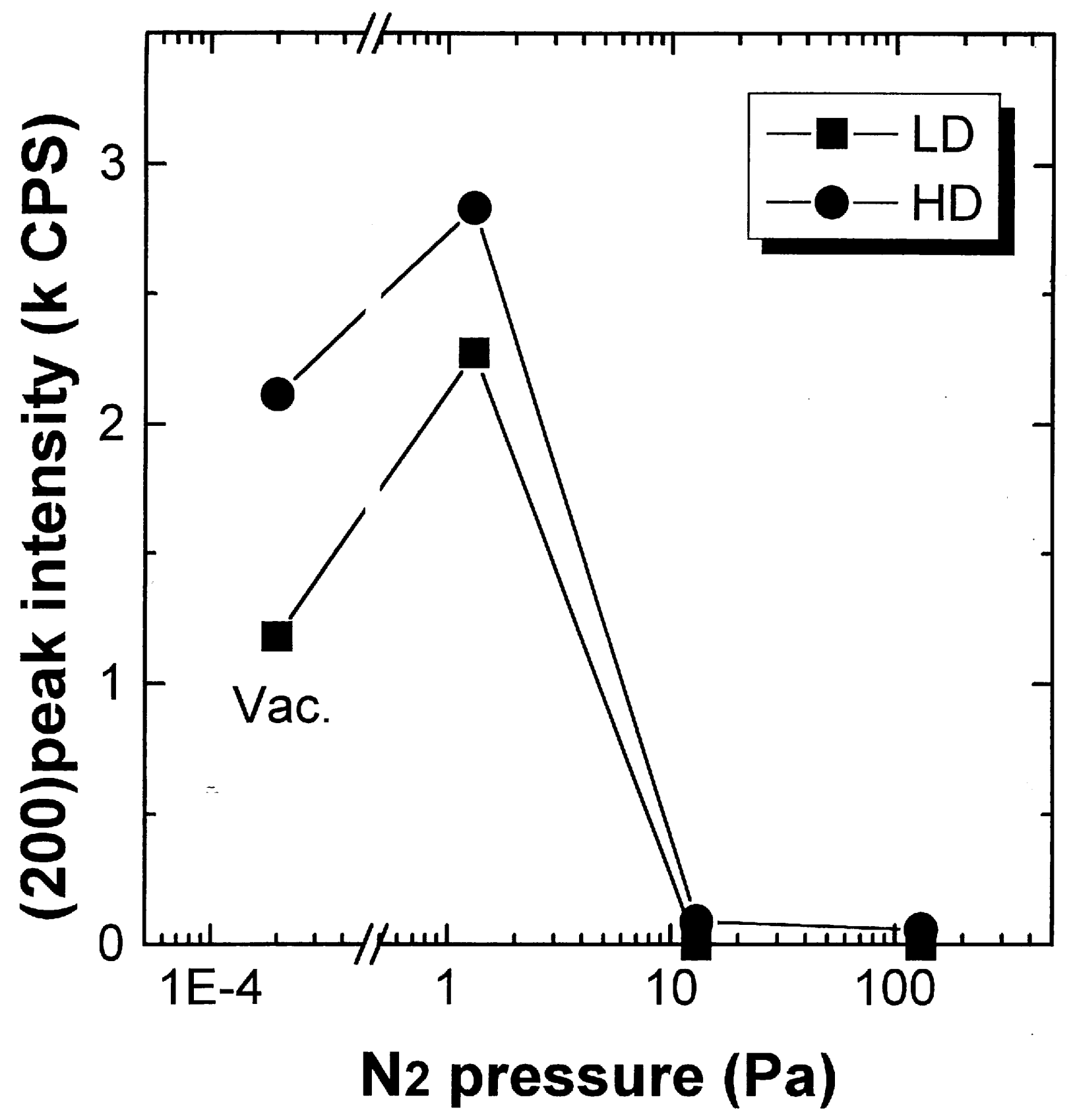

Fig. 2 A Morimoto 


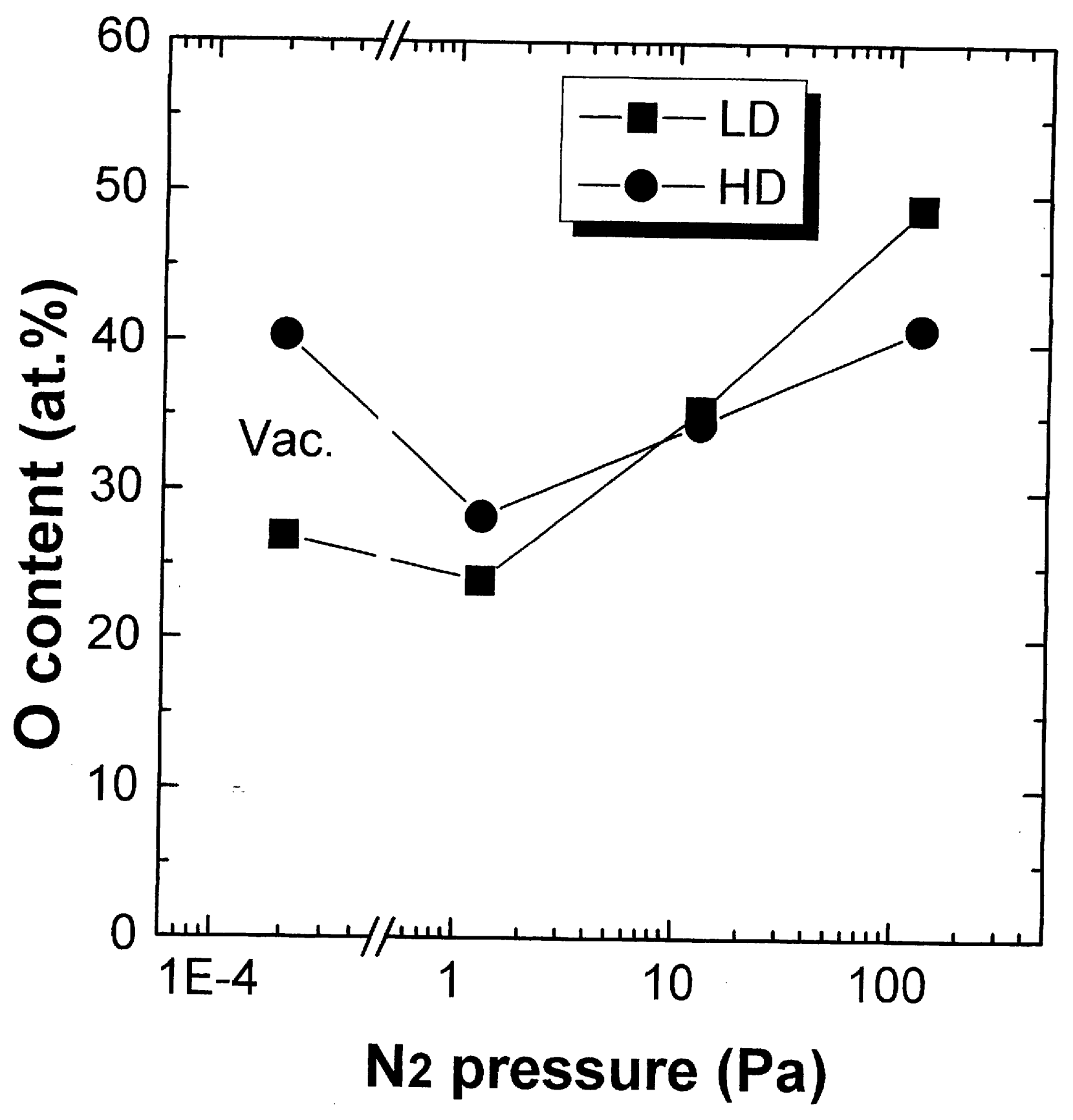

Fig. 3 A Morimoto 


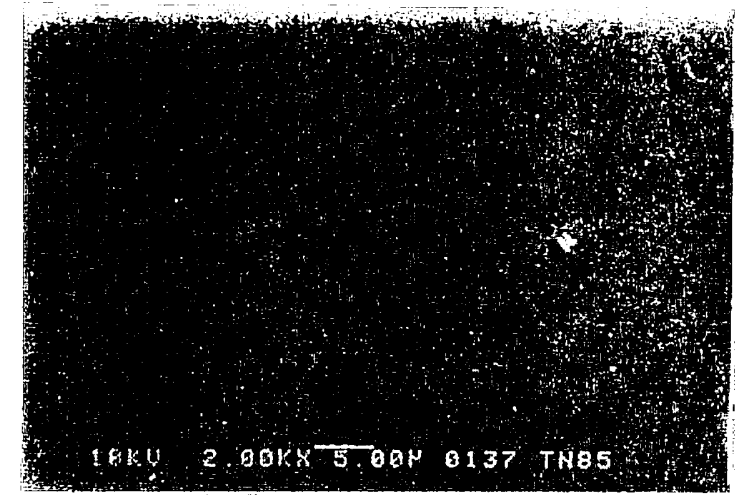

a) Vac.

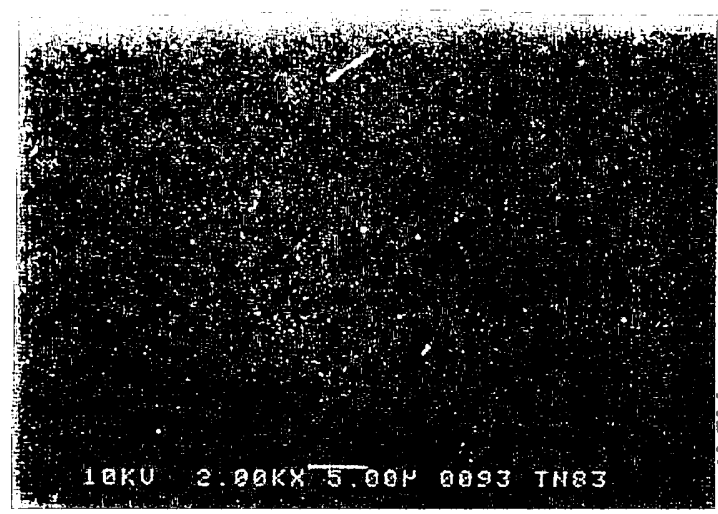

c) $13 \mathrm{~Pa}$

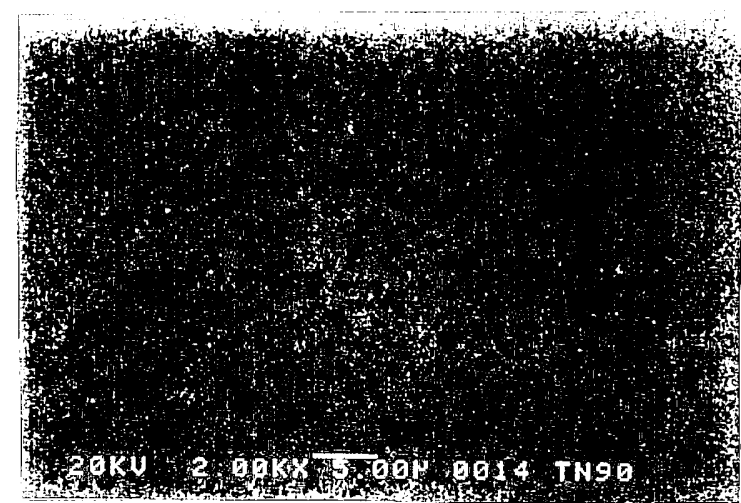

b) $1.3 \mathrm{~Pa}$

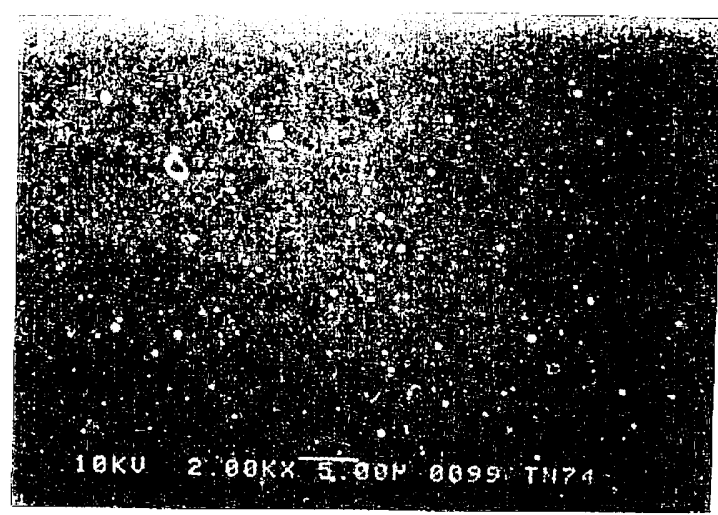

d) $130 \mathrm{~Pa}$

Fig4 A Morimoto 


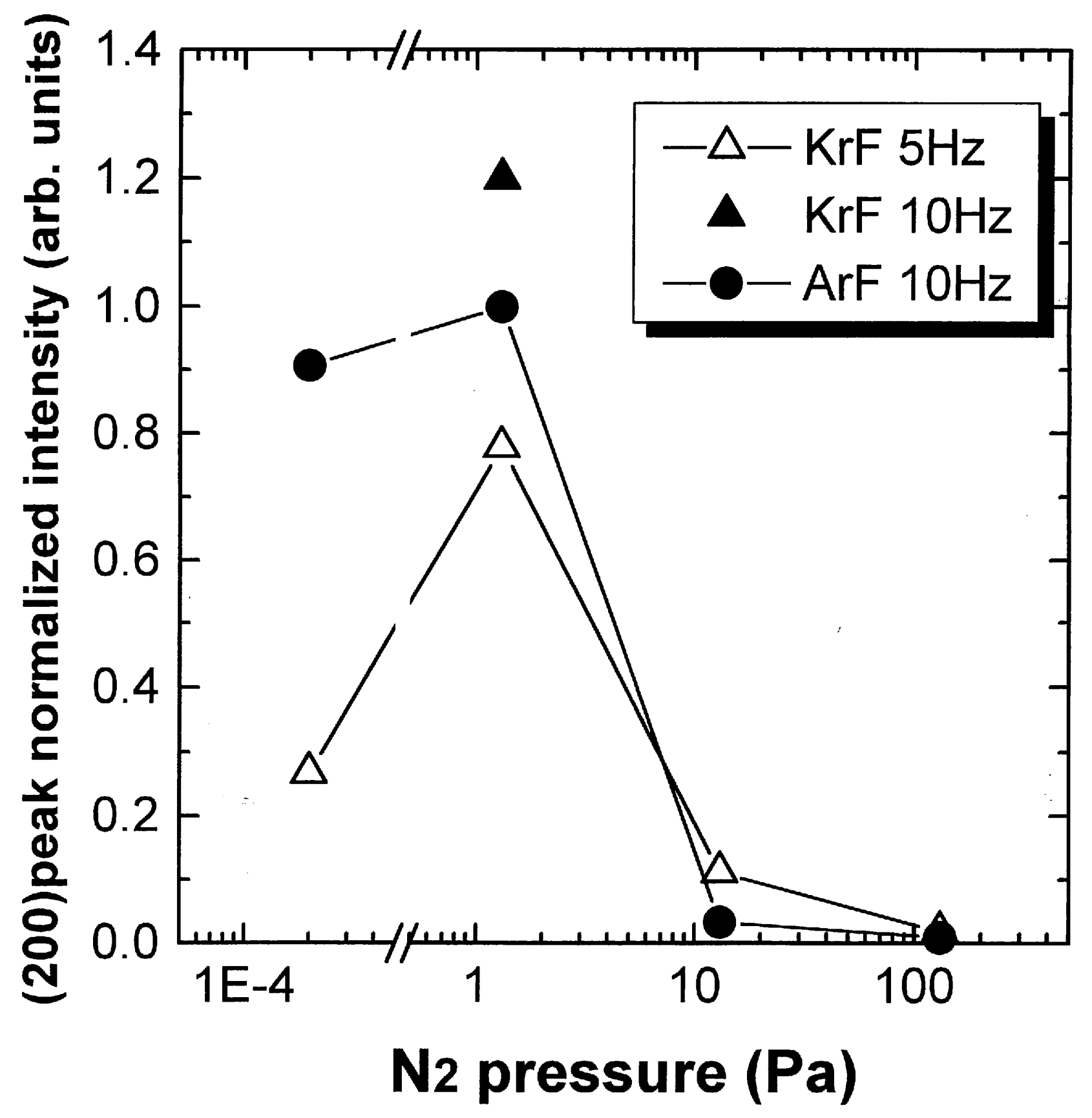

Fiq. 5 A. Morimoto 